\title{
O PAPEL DA GEOGRAFÍA PARA A FORMAÇÃO PROFISSIONAL PARA O SUS FORMAR PARA EL EL SUS: ¿DÓNDE, EN QUÉ, CUÁNTOS?
}

\author{
Luisa Basilia Iñiguez Rojas \\ Universidad de La Habana \\ luisabiniguez@gmail.com
}

Para ser coherentes con la proclama de este simposio, "más SU más geografía", intentaré penetraren el tema que me han asignado, y que agradezco los organizadores. Cuando desde fuera, desde lejos, observamos la trayectoria de la formación de profesionales para el SUS de Brasil, notamos la profusión de esfuerzos que incluyen numerosas instancias, aparatos legales, estrategias políticas que han producido innúmeros documentos organizativos como portarias, relatorios, Programas, cursos con diferentes objetivos y materiales docentes. No hay dudas de que a lo largo de tres décadas se ha hecho MUCHO.

No es mi intención, ni seria ético, valorar que se logró en la formación de profesionales para el SUS, o cuáles son sus deudas, sino a partir de mi Sistema Nacional de Salud, de las experiencias vividas en Brasil, reflexionar en un simposio como este acerca del "donde", con la certeza de que este es substrato esencial que en principio concreta o no, la universalidad, la equidad y la integralidad de cualquiera de nuestros Sistemas de Salud.

\section{¿EN QUÉ Y DÓNDE SE FORMAN?}

La necesidad de profesionales en cualquier Ministerio de Salud, por lo general identifica en primer lugar la formación en medicina, seguida de enfermería, técnicos de enfermería, de técnicos de equipos diagnósticos, de rehabilitación y otros.

Mientras, en las últimas tres décadas, se ha hecho cada vez más frecuente, la formación profesional para la atención primaria o básica en salud. En la actualidad muchos de los esfuerzos se dirigen a la renovación pedagógica como vía para formar profesionales con conocimientos y habilidades que respondan a las exigencias de los principios de los Sistemas de Salud, con prioridad a las estrategias de Salud de la Familia o comunitaria. En varias partes del mundo, con carencias de profesionales sanitarios o no, se avanza en la formación de trabajadores comunitarios, agentes comunitarios, promotores de salud, agentes sanitarios, brigadistas sanitarios, o cualquier otra denominación, que multiplican los actores que atiendan las necesidades de salud de las poblaciones, en algunos casos de forma voluntaria.

En el caso de la formación médica se reitera la necesidad de ampliar el principio docente asistencial enfocado a la vinculación de los estudiantes a las unidades y centros de salud primaria, o a estructuras comunitarias, y otras organizaciones de participación social del sistema de salud, entre otros, fortalecer los departamentos de medicina de la familia en las Facultades de Medicina para incrementar el interés de los estudiantes por la especialidad de medicina familiar, entre otros. (Rossenbatt 1993, Campos 1999).

Según el Censo de Educación Superior del 2017², en el área general de formación denominada "Salud y Bienestar Social" se registran 1088 instituciones, 949 de ellas privadas. Un 17\% del total de cursos ofertados eran de instituciones públicas, con un porciento similar de matrícula y concluyentes En la formación médica las instituciones privadas ofrecían cerca del $60 \%$ del total de cursos, las

Recebido em: 13/10/2019

Aceito para publicação em: 11/11/2019

Palestra proferida no IX Simpósio Nacional de Geografia da Saúde, Blumenau, SC, 2019.

2Instituto Nacional de Estudos e Pesquisas Educacionais Anísio Teixeira - INEP. Censo da Educação Superior no Brasil 2017. portal.mec.gov.br/docman/setembro-2018...censo-superior.

DOI:http://dx.doi.org/ 10.14393/Hygeia153351671 Hygeia 15 (33): 77 - 82, Set/2019 página 77


matrículas y concluyentes superaban ligeramente este valor, que para la formación de odontólogos fue de cerca de un $80 \%$, y para la carrera de farmacia llegó a un $85 \%$. $^{3}$

De forma particular los Programas o cursos generales en salud y en salud pública muestra una interesante distribución, en cuanto los comprendidos como cursos generales en salud como la educación física y la naturología, tienen un total predominio de ofertas y matrículas en instituciones privadas, el único curso de graduación que se reporta en Salud Pública, pertenece a una Universidad pública que en el 2017 ofertó 40 plazas (vagas), para cerca de 300 inscritos. Otros cursos de graduación en salud colectiva, gestión en salud y gestión en salud ambiental, se desarrollan en varias universidades federales del país ${ }^{4}$. Para el resto de las carreras comprendidas en el área se observa un patrón similar de predominio de la formación en instituciones privadas, con la única excepción de la terapia ocupacional que se acerca al $50 \%$.

Los datos expuestos según este registro, muestran el peso de las instituciones privadas en la formación de profesionales en cursos de graduación, y más allá del ranking, que coloca a la mayoría de estas universidades en posiciones inferiores, o de la base social de los estudiantes, lo más importante sería, en ambos tipos de instituciones que para los que estudian medicina por vocación, representación social o por las ventajas del mercado de trabajo, los salarios, o todos estos motivos unidos, procurar como "ideal", una grade curricular y cargas horarias de docencia y asistencia médica social, que fomente el compromiso "por servir al SUS".

Por otra parte, sin datos concretos considero que los cursos, entrenamientos, u otros tipos de capacitación para el SUS en los últimos treinta años, no pueden contarse, y deben haber formado cientos de miles de profesionales en estudios de pos graduación, de nivel medio, hasta incluso haber capacitado población sin nivel de escolaridad terminado. Me atrevo a asegura que la mayoría fueron formados en o por instituciones públicas, federales, estatales o municipales.

Parece evidente que la prioridad está en la formación para la atención básica, la reiterada puerta de entrada al Sistema de atención y donde el $70^{\circ}$ el $80 \%$ de la población encuentra alguna solución a sus problemas de salud, o aprende como cuidar de ella.

\section{¿CUÁNTOS FORMAR PARA ACTUAR DÓNDE?}

Las variantes de las múltiples formaciones que precisa el SUS, llevan implícitas el donde, que pudieran sintetizarse en cuantos para instituciones de tercer y segundo nivel de atención, cuantos para el primer nivel en municipios o comunidades. Cuantos profesionales precisaría el SUS, para atender de forma justa las necesidades de su población, se debate en primer lugar entre voluntad política y financiamiento. De todos es conocido que real o pretexto el financiamiento para el sistema de salud es casi siempre identificado como el principal impedimento para el logro de sus objetivos.

El indicador normal, básico o necesario de médicos por habitantes, pudiera definirse a escala país, o hasta en estados como en el caso de Brasil, pero es claro que la cifra no habla de la "cobertura universal" y aun cuando se halla calculado el indicador que considere las necesidades de toda la población, barreras asociada a la accesibilidad en términos distancia costo, o culturales, en cualquier de nuestros países, puede provocar que la accesibilidad a los servicios sea solo potencial.

\footnotetext{
${ }^{3}$ Cabe destacar que el programa "más médicos", concibió que el $66 \%$ del total de plazas autorizadas para la formación médica fuese en instituciones privadas.

4 Universidade Federal do Rio Grande do Sul, Universidad Federal de Bahia, Universidad Federal do Mato Grosso y Universidad Federal de Rio de Janiero : Curso de graduação em Saúde Coletiva - 4 anos: Universidade Federal do Recôncavo da Bahia: Curso de graduação em Saúde - 3 anos Universidade Federal de Minas Gerais: Curso de graduação de Gestão em Saúde - 4 anos e meio Universidade Federal de Uberlândia: Curso de graduação de Gestão em Saúde Ambiental - 4 anos
} 
En material que trata el primer año del Programa Mas Médicos ${ }^{5}$, se colocaba el indicador desfavorable de cobertura de profesionales médicos en Brasil con 1,8 médicos por 1000 habitantes:

\begin{abstract}
"Brasil precisa de médicos... os médicos por habitantes abaixo da média da OCDE. Para atingir 2,7 médicos, hoje são necessários mais 168.424 médicos". ..700 municípios não têm sequer 1 médico residindo no municipio, 22 estados estão abaixo da média nacional"
\end{abstract}

La meta trazada asumió como referencia el indicador del Reino Unido dado que, "depois do Brasil, tem o mais populoso sistema de saúde pública com características de universalidade". Los dos criterios de comparación parecen de inicio adecuados, no obstante, Brasil tiene una superficie 35 veces mayor que el Reino Unido, con superficie similar al Estado de São Paulo e indicadores económicos y sociales bien alejados de este, entre otros contrastes.

En enero del 2018, informe de la demografía médica6 divulgó que Brasil había alcanzado 452, 801 médicos, para un indicador de 2,18 médicos por mil habitantes. ¿La primera pregunta que podría formularse es que significó este incremento para el SUS de dónde y también de quiénes?

Por otra parte, es posible una previsión aproximada de la necesidad de formación de profesionales para la atención primaria en salud, a la luz de la Política Nacional de Atención Básica de Salud del 2017 publicada en la "portaria 2436 del 2017", y la operacionalización en anexo, tanto la de profesionales médicos, enfermeros, como de agentes comunitarios de salud. En el documento se recomienda entre 2000 y 3500 personas la cantidad de población ser atendida por el médico y la enfermera oscila. Si tomando la cifra de 3000 , para la población actual del país, se necesitarían cerca de 70000 médicos y enfermeras para las unidades básicas, el 15\% de la cifra actual de médicos que se reportan.

En tanto el número de agentes comunitarios en salud, tendría una microárea de atención cuya población no debería exceder las 750 personas, lo que de completarse la cobertura de este integrante del equipo con el máximo de población a atender daría una cifra aproximada de 277000 agentes. No obstante aunque las cifras de personas a atender son "recomendadas", se deja expuesto que esta puede ser mayor o menor según especificidades del territorio y el número de agentes comunitarios de salud y agentes comunitarios de endemias, se definiría según la base poblacional (criterios demográficos, epidemiológicos y socio económicos).

En el documento citado no se hace alguna alusión a cantidad de familias a atender, como había sido propuesto en otros momentos, y evidente claro que los profesionales para la atención básica, deben formarse en temas sociológicos y psicológicos asociados a la salud familiar.

Las cifras citadas, dan la medida de los esfuerzos que se precisan para la formación de recursos que logren los objetivos que se propone la estrategia de atención básica en el país. Más aún cuando en la composición y normativas de funcionamiento de los equipos de atención básica en sus varios tipos, se coloca la "preferencia "por médicos y enfermeras o auxiliares de enfermería formados en la especialidad de medicina de la familia y comunidad".

Por otra parte, también en el documento de Política Nacional de Atención Básica se define que el agente comunitario en salud y el agente comunitario de endemias, además de las atribuciones comunes a todos los miembros del equipo tendrá la capacidad de: Realizar un diagnóstico demográfico, social, cultural, ambiental y epidemiológico del territorio en que actúan, contribuyendo para el proceso de territorialización y mapeamiento del área de actuación del equipo de atención básica. Aunque loable, parece poco probable, que sin una capacitación específica, los agentes logren completar este diagnóstico con la profundidad requerida. Al respecto existen múltiples ejemplos de experiencias desarrolladas por instituciones del Ministerio de Salud y departamentos de universidades, durante décadas encaminadas a este tipo de formación de agentes.

\footnotetext{
${ }^{5}$ Ministerio de Saúde. Mais Medicos. 1 ano. Presentado al V Congreso Internacional de Geografía de la Salud. 2014

$6 \quad$ http://www.portal.cfm.org.br/index.php?option=com content\&view=article\&id=27503:2018-03-19-19-44$32 \&$ catid $=3$
} 


\section{¿FORMAR PARA QUE, CUÁNTOS, PARA DÓNDE?}

Prever la necesidad de formar una determinada cantidad de profesionales médicos según especialidades para territorios nacionales o sub nacionales, tal vez ni sea una pregunta "normal" en un Sistema de Salud, menos aún cuando coordinado o no, se desarrolla en paralelo la formación por parte de instituciones privadas.

Es difícil imaginar un sistema que consiga calcular la necesidad de formación de un número de cardiólogos, ortopédicos, dermatólogos y decenas de otros especialistas para el país y consiga asegurarla. Más complicado sería este cálculo para sus unidades políticas administrativas sub nacionales, aún con un sistema regionalizado?

Todavía más difícil es imaginar como un Sistema de Salud pudiera conocer que parte de los formados cada año, se dispondrían a permanecer en el SUS después de cumplir su servicio social, si procede, o irían al servicio exclusivamente privado, en clínicas o a sus propios consultorios.

El incremento en cinco años de 1,8 a 2,18 médicos por 1000 habitante, debe ser resultado en primer lugar de graduaciones más elevadas, no obstante es posible valorar ese incremento en términos de cumplimiento de los principios del SUS, o de la mejora de la salud de la población de ¿dónde?

En la portaría antes citada, se destaca que la cobertura en términos territoriales continúa con grandes desigualdades, elevada concentración de médicos en las capitales de la mayoría de los estados y muy baja cobertura o carencia de atención en municipios con dificultades de acceso, dispersión de la población, donde no se logran fijar los profesionales, por la falta de políticas territoriales lo que a su vez provocan el "silencio epidemiológico". ${ }^{8}$

En una de las tablas que muestra la distribución de médicos por regiones según número y cantidad de población de los municipios, incluye la razón de médicos por 1000 habitante. Un simple cálculo halla que en 4601 municipios (83\% del total) la cobertura es de menos de 1 médico por 1000 habitantes, y se incluyen tanto aquellos que contienen entre 50000 y 1000000 habitantes en las regiones norte y nordeste, o hasta 20000 habitantes en las regiones sur y sudeste.

De inicio parece sorprender, poco después aparece una inquietud. Menos de un médico por 1000 habitantes puede significar un verdadero desastre en términos de atención, o ser suficiente para determinados contextos territoriales, barriales. Por otra parte, la política nacional de atención básica como fue expuesto propone un médico por unidad de entre 2000 y 3500 personas, sin alusión a la distribución de este, componente decisivo de la accesibilidad no solo a la unidad de salud de la familia, como a otros servicios donde serán encaminados.

La pertinencia podría pensarse en primer lugar en los términos más tradicionales de la heterogeneidad territorial, lo urbano y lo rural. Como en cualquier país, las divergencias entre necesidades y formación de recursos humanos es aún más complicado en territorios rurales.

En la heterogeneidad del "rural" brasilero pudiera considerarse según una de las propuestas de clasificación ${ }^{9}$ formar para el rural indígena, rural riberinho, rural perirubano, rural de polos agrícolas, o formar como una reciente propuesta de IBGE en el rural adyacente y el rural remoto. Es evidente que cualquiera de estos "rurales", no tiene las mismas necesidades de salud que el rural remoto de la región norte, como no la tienen barrios "vecinos distantes" por sus condiciones materiales de vida, nivel educacional, aspiraciones, distantes, más allá de las clásicas clases sociales.

En artículo titulado Está na hora do treinamento em saúde rural para médicos de família no Brasil! Almeida et al. $2018^{10}$ hacen un "apelo urgente" para el debate sobre las necesidades de la población

\footnotetext{
7 La aproximación a cuantos se apoyaría en el sistema de referencia y contra referencia del nivel básico de atención, básica, o de la cantidad de consultas y otros atendimientos de urgencia, pero difícilmente podría cuantificar la demanda oculta de atención especializada. Las informaciones que además varían según las dinámicas socio demográficas.

${ }^{8}$ No hay daños a la salud, porque no hay quien los registre.

${ }^{9}$ tipologia regionalizada dos espaços rurais brasileiros - DPI/INPE www.dpi.inpe.br/.../JanBitoun et al TIPOLOGIA TX FINAL REVISADOJB2.pdf ${ }^{10}$ https://www.rbmfc.org.br/rbmfc/article/view/1696/902
} 
rural y posibles modelos para proyectar la provisión de recursos para atender la salud en estos territorios.

En la presentación del suplemento de la Revista de Salud Pública dedicado a la salud rural, con el título "Salud rural en Brasil: tema antiguo más que actual"11, Coimbra coloca que en el Brasil contemporáneo se alcanzaron logros en el campo de la educación y la salud rural, que consiguieron controlar varias enfermedades infecciosas y parasitarias, pero, en la actualidad poco se sabe de los desafíos que enfrentan estas poblaciones para mantener su salud, o su seguridad alimentaria.

Compreender por que a situação de saúde das populações rurais "está assim" é um passo fundamental para a concepção e implementação de políticas públicas mais adequadas para um Brasil que, mesmo nas regiões mais investigadas e conhecidas, como no Sul, ainda permanece bastante desconhecido e negligenciado.

A propósito en la Política Nacional de Atención Básica de Salud antes citada se expone como sería la formación de equipos de atención básica para cuatro poblaciones específicas: "Equipe de atenção básica prisional con una distribución territorial irregular y el Equipe de consultorio de rua" localizado en áreas urbanas. Los Equipos de salud de la familia riberiña se dividen en dos opciones organizativas, con requerimientos e incentivos financieros para apoyos logísticos diferentes": Equipes de saúde da familia para Populãçao Riberinha da Amazonia Legal y Pantaneiras y Equipes de Saúde da familia fluviais.

"Lo rural" aparece solo de forma implícita solo en los estos dos equipos expuestos, lo que sin restarles importancia, parece insuficiente. ¿No debería haber unidades de atención básica, que optaran por financiamientos específicos para territorios de poblaciones indígenas, o rurales en territorios de la seca, o en barrios precarios de las capitales?.

Para cualquier tipo de formación que apoye el sistema de salud, lo óptimo "ideal" pudiera ser formar acorde a la diversidad ambiental, cultural, económica y social, que en países como Brasil, llegaría a regiones o a estados, pero también a contextos municipales, teniendo en cuenta la existencia de cursos de graduación en relativamente pequeñas ciudades del interior de varios estados. Sea cual fuere, el donde, para que, la pertinencia de cuantos, sería siempre una interrogación, proyectada entre intereses, ni siempre compatibles con los principios del SUS, y que variaría en el tiempo según las dinámicas socio demográficas de los territorios.

\section{REFLEXIONES FINALES}

Tal vez más que la falta de médicos de Brasil, lo que hoy más preocupa es la discordante relación ente la formación de recursos humanos y su distribución. Brasil es un país con desafíos enormes para el logro de la equidad en la dotación de cualquiera de los servicios. Aunque se alude casi siempre a sus dimensiones continentales, otros componentes de su complejidad, pueden ser aún más importantes como la fragmentación municipal, la heterogénea distribución de la población regulada por sus regiones naturales o ecológicas (selva, sertões, montañas... ), la historia de la ocupación y los procesos de concentración en ciudades.

No obstante, el peso de políticas nacionales, o en algunos casos su ausencia, o implementación fallida, impugna los principios del SUS, a lo cual como país federativo, se integra la "gestión autónoma" de recursos generados en los estados, que aporta diferencias en la eficiencia y la equidad de los servicios de salud.

Al asumir estos presupuestos, no quedarán dudas de que la formación de profesionales para el SUS brasilero, a pesar de políticas normativas, ha tomado y tomará caminos difíciles, hasta un tiempo hoy indeterminado, cuando se cambien "mentalidades" que permitan saldar deudas materiales y éticas en favor del bienestar y la salud de su población. Me atrevería a decir, sin fanatismos, que más geografía

\footnotetext{
${ }^{11} \mathrm{http}: / / \mathrm{www}$. scielo.br/scielo.php?script=sci arttext\&pid=S0034-89102018000200200\&lng=pt\&nrm=iso\&tlng=pt
} 
comprometida en este periodo popular de la historia como quisiera Milton Santos, sería una de las vías para lograrlo, no necesariamente quiere decir más geógrafos.

Concebir la formación profesional para el SUS" tendría como perfil ideal, el conocimiento del territorio de actuación y las condiciones de reproducción social de las familias que en el residen, la capacidad de identificar riesgos, organizar acciones, y conocimientos básicos de salud pública, sociología, antropología, epidemiologia, geografía, cartografía, biología, ecología, medicina y un compromiso

Son hechos bien conocidos que falta coherencia entre la formación de los profesionales del sistema y sus principios rectores, que fueran definidos, incluso antes de su constitución oficial y que falta pertinencia en la formación de profesionales, y en especial, pertinencia en términos de distribución territorial.

Las preguntas del título de esta intervención, no consideraron la que tal vez sea la más importante. ¿Formar a quienes?, En este sentido debe recordarse que entre tantos objetivos del movimiento sanitarista de Brasil hace más de 30 años, se incluyó formar a los activistas políticos, a la vanguardia política, que aún con poco poder, o sin ninguno, consiguiera forzar una Reforma Sanitaria que perseguía más allá de la descentralización de los servicios, de la participación dela población en las decisiones, el derecho universal a la salud, con mejores condiciones de vida para el pueblo.

No quedan dudas de que la vanguardia política debe ser multiplicada, el sentimiento de pertenecer al SUS tiene que fomentarse sin descanso, $Y$ tengo certeza de que este simposio está consciente de ello. Formar agentes comunitarios y ciudadanos para apoyar el lastimado Sistema de Salud sería el camino más acertado, pero solo contar con la valentía, 